\title{
Performance Evaluation of Image Segmentation Method based on Doubly Truncated Generalized Laplace Mixture Model and Hierarchical Clustering
}

\author{
T.Jyothirmayi \\ Department of Computer Science and Engineering, GITAM University, Visakhapatnam \\ Email: koka.jyothirmayi@gmail.com
}

K.Srinivasa Rao

Department of Statistics, Andhra University, Visakhapatnam

Email: ksrao@yahoo.co.in

P.Srinivasa Rao

Department of Computer Science and Systems Engineering, Andhra University, Visakhapatnam

Email: peri.srinivasarao@yahoo.com

Ch.Satyanarayana

Department of Computer Science and Engineering, JNTU-Kakinada

Email: chsatyanarayana@yahoo.com

\begin{abstract}
The present paper aims at performance evaluation of Doubly Truncated Generalized Laplace Mixture Model and Hierarchical clustering (DTGLMM$\mathrm{H})$ for image analysis concerned to various practical applications like security, surveillance, medical diagnostics and other areas. Among the many algorithms designed and developed for image segmentation the dominance of Gaussian Mixture Model (GMM) has been predominant which has the major drawback of suiting to a particular kind of data. Therefore the present work aims at development of DTGLMM-H algorithm which can be suitable for wide variety of applications and data. Performance evaluation of the developed algorithm has been done through various measures like Probabilistic Rand index (PRI), Global Consistency Error (GCE) and Variation of Information (VOI). During the current work case studies for various different images having pixel intensities has been carried out and the obtained results indicate the superiority of the developed algorithm for improved image segmentation.
\end{abstract}

Index Terms-Image segmentation, Generalized Laplace Mixture Model, doubly truncated generalized Laplace Mixture Model, EM algorithm.

\section{INTRODUCTION}

Image segmentation aims at identifying the regions of interest in an image or annotating the data of an image. It is the process to classify an image into several clusters according to the feature of image. Image segmentation techniques are based on some pixel or region similarity measures in relation to their local neighborhood. Segmentation techniques are broadly classified as region based, edge based, threshold based and model based [14]. Among these model based segmentation algorithms are found to be efficient compared to other [5]. In model based, entire image is viewed as a collection of image regions and each image region is characterized by a probability distribution function of pixels.

The pixel intensity is considered as a feature component of the image. The pixel intensities in image may be meso kurtic, platy kurtic, lepto kurtic, symmetric and asymmetric. The efficiency of segmentation algorithm depends on probability distribution followed by the pixels in an image.

Much work has been reported considering the pixel intensities follow a Gaussian distribution and variates of finite GMM. Yunjie Chen et al[6] analyzed Gaussian Mixture Model based on Non Local Information for brain MR images segmentation. Legendre polynomials were used to fit and merged to the EM framework and non local information was also used to preserve the geometrical edges information. Karim Kalti et al[7] analyzed an image segmentation method based on Gaussian Mixture model and modified FCM Algorithm. The classification was made on the basis of adaptive distance which privileged the one or the other features according to the spatial position of the pixel in the image. Zhaoxia Fu et al [8] proposed an image segmentation method which used Gaussian Mixture Models to model 
the original image and transforms the segmentation problem into maximum likelihood parameter estimation by expectation-maximization(EM algorithm) and classify the pixels in image. The application of GMM is accurate and successful for all types of data except lepto kurtic. To overcome this drawback generalization of Gaussian mixture models with respect to kurtosis is considered. Laplace probability model serves as a alternate to Gaussian distribution with respect to platy kurtic, lepto kurtic and asymmetric data. Srinivasa Rao et al[9] generalized the laplace distribution as generalized Laplace distribution. Jyothirmayi et al[10] later developed and analyzed the generalized Laplace mixture model(GLMM) for image segmentation. In these algorithms the range of pixel intensities was assumed as $\{-\infty, \infty\}$.

The developed model was integrated with hierarchical clustering method and used for image segmentation of images which are having platykurtic and lepto kurtic nature. Hierarchical clustering and moment method of estimation was used for initialization of parameters. As an extension to the previous works, within the current work an attempt is made to extend the GLMM as Doubly Truncated GLMM (DTGLMM) by truncating the range of pixel intensity values within a specified range. Performance evaluation of the developed model has been carried out by means of analysis of various different categories of images as case studies.

\section{PROPOSED WORK}

In this paper an algorithm DTGLMM-H has been proposed for image segmentation. It is assumed that the whole image is collection of image regions in which the pixel intensity of each region follows a generalized laplace distribution. The parameters mean, variance of DTGLMM are estimated through EM algorithm. Initialization of the parameters is obtained by hierarchical clustering. Image analysis with the developed algorithm is performed on five images from Berkeley image data set and compared with existing algorithms available in the literature

\section{A. Doubly Truncated Generalized Laplace Distribution}

An image is considered as a collection of image regions for segmentation algorithms. Each image is quantized by pixel intensities. The pixel intensity $z=f(x, y)$ is a random variable for any given point in image region.
The pixel intensities within the region are assumed to have an infinite range. But in any image the pixel intensity lie between two values. Assuming that the pixel intensity lie between ' $a$ ' and ' $b$ ', the probability density function of the pixel intensity is given by

$$
\begin{gathered}
f\left(x, \mu, \sigma^{2}\right) \\
\left(\frac{\left(r^{2}+\frac{(x-\mu)^{2}}{\sigma^{2}}\right)^{r} e^{-\left|\frac{x-\mu}{\sigma}\right|}}{2 \sigma \sum_{k=0}^{r}\left(\begin{array}{l}
r \\
k
\end{array}\right) r^{2(r-k)}\left(\int_{\frac{b-\mu}{\sigma}}^{\frac{b-\mu}{\sigma}} x^{2 k} e^{-|x|} d x\right)}\right) \\
\text { where a }<x<b, a<\mu<b, \sigma>0
\end{gathered}
$$

This can also be represented as

$$
\begin{aligned}
& f\left(x, \mu, \sigma^{2}\right) \\
& 2 \sigma \sum_{k=0}^{r}\left(\begin{array}{l}
r \\
k
\end{array}\right) r^{2(r-k)}\left[\gamma\left[(2 k+1),-\left(\frac{a-\mu}{\sigma}\right)\right]+\gamma\left[(2 k+1),-\left(\frac{b-\mu}{\sigma}\right)\right]\right]
\end{aligned}
$$

It is supposed that entire image is collection of several image regions where each image region's pixel intensities are characterized by doubly truncated generalized Laplace probability model. The probability density function of pixel intensities in whole image is of the form

$$
p(x)=\sum_{i=1}^{k} \alpha_{i} f_{i}\left(\mathrm{x}_{\mathrm{s}}, \mu_{i},{\sigma_{i}}^{2}\right)
$$

Where $\mathrm{k}$ is the number of regions, $0 \leq \alpha_{\mathrm{i}} \leq 1$ are weights such that $\Sigma \alpha_{\mathrm{i}}=1$ and $\alpha_{\mathrm{i}}$ is the weight associated with $\mathrm{i}^{\text {th }}$ region in the whole image and $f_{i}\left(x, \mu, \sigma^{2}\right)$ is the probability density function of Generalized Laplace distribution of $i^{\text {th }}$ image region and is as given in equation(1)

The mean of the distribution is

$$
\begin{gathered}
\mathrm{E}(\mathrm{X})=\int_{\mathrm{a}}^{\mathrm{b}} \mathrm{xf}(\mathrm{x}) \mathrm{dx}=\mu+ \\
\frac{\sum_{\mathrm{k}=0}^{\mathrm{r}}\left(\begin{array}{l}
\mathrm{r} \\
\mathrm{k}
\end{array}\right) \mathrm{r}^{2(\mathrm{r}-\mathrm{k})}\left[\gamma\left[(2 \mathrm{k}+2),\left(\frac{\mathrm{b}-\mu}{\sigma}\right)\right]-\gamma\left[(2 \mathrm{k}+2),-\left(\frac{\mathrm{a}-\mu}{\sigma}\right)\right]\right]}{\sum_{\mathrm{k}=0}^{\mathrm{r}}\left(\begin{array}{l}
\mathrm{r} \\
\mathrm{k}
\end{array}\right) \mathrm{r}^{2(\mathrm{r}-\mathrm{k})}\left[\gamma\left[(2 \mathrm{k}+1),\left(\frac{\mathrm{b}-\mu}{\sigma}\right)\right]-\gamma\left[(2 \mathrm{k}+1),-\left(\frac{\mathrm{a}-\mu}{\sigma}\right)\right]\right]}
\end{gathered}
$$

The variance of the distribution is

$$
\begin{aligned}
& \operatorname{var}(\mathrm{x})=\operatorname{var}\left(\frac{1}{\mathrm{n}} \sum_{\mathrm{i}=1}^{\mathrm{n}} \mathrm{X}_{\mathrm{i}}\right)= \\
& \frac{\frac{1}{\mathrm{n}} \sum_{\mathrm{q}=0}^{2}\left(\begin{array}{l}
2 \\
\mathrm{q}
\end{array}\right)\left(\mu-\mu_{1}^{1}\right)^{2-\mathrm{q}} \sigma^{\mathrm{q}}\left[\begin{array}{c}
(-1)^{\mathrm{q}} \gamma\left[(\mathrm{q}+1),-\left(\frac{\mathrm{a}-\mu}{\sigma}\right)\right] \\
\left.+(-1)^{\mathrm{q}} \gamma\left[(\mathrm{q}+3),-\left(\frac{\mathrm{a}-\mu}{\sigma}\right)\right]+\gamma\left[(\mathrm{q}+1),-\left(\frac{\mathrm{b}-\mu}{\sigma}\right)\right]+\gamma\left[(\mathrm{q}+3),-\left(\frac{\mathrm{b}-\mu}{\sigma}\right)\right]\right]
\end{array}\right.}{\sum_{\mathrm{k}=0}^{\mathrm{r}}\left(\begin{array}{l}
\mathrm{r} \\
\mathrm{k}
\end{array}\right) \mathrm{r}^{2(\mathrm{r}-\mathrm{k})}\left[\gamma\left[(2 \mathrm{k}+1),-\left(\frac{\mathrm{a}-\mu}{\sigma}\right)\right]-\gamma\left[(2 \mathrm{k}+1),\left(\frac{\mathrm{b}-\mu}{\sigma}\right)\right]\right]}
\end{aligned}
$$




\section{B. Estimation of the Model Parameters By EM Algorithm}

To estimate the model parameters, EM algorithm is utilized by maximizing the expected likelihood function. It is assumed that the intensity of pixel in image region follows a new Laplace distribution and whole image is characterized with a finite mixture of new generalized Laplace distributions. To get accurate result doubly truncated generalized Laplace distribution is well suited where range of pixel intensities is assumed to be finite. Its probability distribution function is given in equation 2 .

The likelihood function of observations $\mathrm{x}_{1}, \mathrm{x}_{2} \ldots \mathrm{x}_{\mathrm{n}}$ is

$$
\begin{gathered}
\mathrm{L}(\theta)=\prod_{s=1}^{N} p\left(\mathrm{x}_{s}, \theta^{l}\right) \\
\text { (i.e) } \mathrm{L}(\theta)=\prod_{s=1}^{N}\left(\sum_{i=1}^{k} \alpha_{i} f_{i}\left(\mathrm{x}_{\mathrm{s}}, \mu_{i}, \sigma_{i}{ }^{2}\right)\right)
\end{gathered}
$$

$\log \mathrm{L}(\theta)=\sum_{s=1}^{N} \log \left(\sum_{i=1}^{k} \alpha_{i} f_{i}\left(\mathrm{x}_{\mathrm{s}}, \mu_{i}, \sigma_{i}^{2}\right)\right)$

where

$$
\begin{gathered}
\theta=\left\{\mu, \sigma^{2}, \alpha_{i} ; \mathrm{i}=1,2, . . \mathrm{k}\right\} \\
\log \sum_{s=1}^{N} \log \left[\left(\frac{\left(r^{2}+\frac{(x-\mu)^{2}}{\sigma^{2}}\right)^{r} e^{-\left|\frac{x-\mu}{\sigma}\right|}}{2 \sigma \sum_{k=0}^{r}\left(\begin{array}{c}
r \\
k
\end{array}\right) r^{2(r-k)}\left(\int_{\frac{a-\mu}{\sigma}}^{\frac{b-\mu}{\sigma}} x^{2 k} e^{-|x|} d x\right)}\right)\right]
\end{gathered}
$$

E Step: In the E Step the expectation value of $\log \mathrm{L}(\theta)$ with respect to initial parameter $\theta^{0}$ is

$$
\begin{gathered}
\mathrm{Q}\left(\theta ; \theta^{0}\right)=E_{\theta^{0}}[\log L(\theta)] \\
\mathrm{P}\left(x_{S}, \theta^{\mathrm{l}}\right)=\sum_{\mathrm{i}=1}^{\mathrm{k}} \alpha_{\mathrm{i}}^{\mathrm{l}} \mathrm{f}_{\mathrm{i}}\left(\mathrm{x}_{\mathrm{S}}, \theta^{\mathrm{l}}\right)
\end{gathered}
$$$$
\log \mathrm{L}(\theta)=\sum_{\mathrm{s}=1}^{\mathrm{N}} \log \left(\sum_{\mathrm{i}=1}^{\mathrm{k}} \alpha_{\mathrm{i}}^{\mathrm{l}} \mathrm{f}_{\mathrm{i}}\left(\mathrm{x}_{\mathrm{s}}, \theta^{\mathrm{l}}\right)\right)
$$
is

The conditional probability of $\mathrm{x}_{\mathrm{s}}$ belonging to region $\mathrm{k}$

$$
\mathrm{T}_{\mathrm{k}}\left(\mathrm{x}_{\mathrm{s}}, \theta^{\mathrm{l}}\right)=\frac{\alpha_{\mathrm{k}} \mathrm{f}_{\mathrm{k}}\left(\mathrm{x}_{\mathrm{s}}, \theta^{\mathrm{l}}\right)}{\mathrm{p}\left(\mathrm{x}_{\mathrm{s}}, \theta^{\mathrm{l}}\right)}=\frac{\alpha_{\mathrm{k}} \mathrm{l}_{\mathrm{k}}\left(\mathrm{x}_{\mathrm{s}} \theta^{\mathrm{l}}\right)}{\sum_{\mathrm{i}=1}^{\mathrm{k}} \mathrm{a}_{\mathrm{i}} \mathrm{f}_{\mathrm{i}}\left(\mathrm{x}_{\mathrm{s}}, \theta^{\mathrm{l}}\right)}
$$

$\mathrm{Q}\left(\theta ; \theta^{0}\right)=\sum_{\mathrm{i}=1}^{\mathrm{k}} \sum_{\mathrm{s}=1}^{\mathrm{N}} \mathrm{T}_{\mathrm{i}}\left(\mathrm{x}_{\mathrm{s}}, \theta^{\mathrm{l}}\right)\left(\log \mathrm{f}_{\mathrm{i}}\left(\mathrm{x}_{\mathrm{s}}, \theta^{\mathrm{l}}\right)+\log \alpha_{\mathrm{i}}^{\mathrm{l}}\right.$

Where

$$
\mathrm{f}_{\mathrm{i}}\left(\mathrm{x}_{\mathrm{S}}, \theta^{\mathrm{l}}\right)=\left(\frac{\left(r^{2}+\frac{(x-\mu)^{2}}{\sigma^{2}}\right)^{r} e^{-\left|\frac{x-\mu}{\sigma}\right|}}{2 \sigma \sum_{k=0}^{r}\left(\begin{array}{l}
r \\
k
\end{array}\right)^{2(r-k)}\left(\int_{\frac{a-\mu}{\sigma}}^{\frac{b-\mu}{\sigma}} x^{2 k} e^{-|x|} d x\right)}\right)
$$

M Step: To get estimation of parameters, maximize $\mathrm{Q}\left(\theta ; \theta^{l}\right)$ such that $\sum \alpha_{\mathrm{i}}=1$.

Using Lagrange type function and maximizing

$$
\widehat{\alpha}_{l}=\frac{1}{\mathrm{~N}} \sum_{\mathrm{s}=1}^{\mathrm{N}} \mathrm{T}_{\mathrm{i}}\left(\mathrm{x}_{\mathrm{s}}, \theta^{\mathrm{l}}\right)
$$

$\alpha_{\mathrm{i}}$ for $(1+1)$ th iteration is

$$
\alpha_{i}{ }^{1+1}=\frac{1}{N} \sum_{s=1}^{\mathrm{N}} T_{i}\left(x_{s}, \theta^{l}\right)=\frac{1}{N} \sum_{s=1}^{N}\left[\frac{a_{i} f_{i}\left(x_{s}, \theta^{1}\right)}{\sum_{i=1}^{k} a_{i} f_{i}\left(x_{s}, \theta^{l}\right)}\right]
$$

For updating the parameter $\mu_{\mathrm{i}}, \mathrm{i}=1,2, . \mathrm{k}$

Consider the derivative of $\mathrm{Q}\left(\theta ; \theta^{l}\right)$ with respect to $\mu_{\mathrm{i}}$, and equate to 0

$$
\begin{gathered}
\mathrm{Q}\left(\theta ; \theta^{l}\right)=\mathrm{E}\left[\log \mathrm{L}\left(\left(\theta ; \theta^{l}\right)\right]\right. \\
\frac{\partial}{\partial \mu \mathrm{i}} \mathrm{Q}\left(\theta ; \theta^{l}\right)=0
\end{gathered}
$$

This implies

$$
\begin{aligned}
& \frac{\partial}{\partial \mu \mathrm{i}}\left[\sum_{\mathrm{i}=1}^{\mathrm{k}} \sum_{s=1}^{\mathrm{N}} \mathrm{T}_{\mathrm{i}}\left(\mathrm{x}_{s}, \theta^{l}\right)\left[\log \left[\left(\frac{\left(r^{2}+\frac{(x-\mu)^{2}}{\sigma^{2}}\right)^{r} e^{-\frac{x-\mu^{-} \mid}{\sigma}}}{2 \sigma \sum_{k=0}^{r}\left(\begin{array}{l}
r \\
k
\end{array}\right) r^{2(r-k)}\left(\int_{\frac{b-\mu}{\sigma}}^{\frac{b-\mu}{\sigma}} x^{2 k} e^{-|x|} d x\right)}\right)\right]\right]\right. \\
& \left.+\log \left(\alpha_{\mathrm{i}}^{1}\right)\right]=0
\end{aligned}
$$

This implies

$$
\begin{aligned}
& \frac{\partial}{\partial \mu \mathrm{i}}\left[\sum _ { \mathrm { i } = 1 } ^ { \mathrm { k } } \sum _ { s = 1 } ^ { \mathrm { N } } \mathrm { T } _ { \mathrm { i } } ( \mathrm { x } _ { \mathrm { s } } , \theta ^ { l } ) \left[\log \left[\left(\left(r^{2}+\frac{\left(\mathrm{x}_{\mathrm{s}}-\mu_{\mathrm{i}}\right)^{2}}{\sigma_{i}^{2}}\right)^{r}\right)\right]\right.\right. \\
& -1 / 2\left|\frac{x_{s}-\mu_{\mathrm{i}}}{\sigma_{i}}\right|-\log \left(\sigma_{i}\right) \\
& \left.-\log \left(\sum_{k=0}^{r}\left(\begin{array}{l}
r \\
k
\end{array}\right) r^{2(r-k)} \int_{\frac{a-\mu}{\sigma}}^{\frac{b-\mu}{\sigma}} x^{2 k} e^{-|x|} d x\right)\right]+\log \left(\alpha_{\mathrm{i}}{ }^{\mathrm{l}}\right) \\
& =0
\end{aligned}
$$

This implies

$$
\begin{gathered}
\sum_{\mathrm{s}=1}^{\mathrm{N}} \mathrm{T}_{\mathrm{i}}\left(\mathrm{x}_{\mathrm{s}}, \theta^{\mathrm{l}}\right)\left[( \frac { ( \mathrm { x } _ { \mathrm { s } } - \mu _ { \mathrm { i } } ) } { \sigma _ { i } ^ { 2 } } ) \left[\frac{\left[-2 \sigma_{i}^{2}\right]}{\mathrm{r}^{2} \sigma_{i}^{2}+\left(\mathrm{x}_{\mathrm{s}}-\mu_{\mathrm{i}}\right)^{2}}\right.\right. \\
\left.\left.+\frac{\sigma_{\mathrm{i}}}{\left|\mathrm{x}_{\mathrm{s}}-\mu_{\mathrm{i}}\right|}\right]\right]=0 \\
\sum_{\mathrm{s}=1}^{\mathrm{N}} \mathrm{T}_{\mathrm{i}}\left(\mathrm{x}_{\mathrm{s}}, \theta^{\mathrm{l}}\right) \frac{\mathrm{x}_{\mathrm{s}}-\mu_{\mathrm{i}}}{\sigma_{\mathrm{i}}\left|\mathrm{x}_{\mathrm{s}}-\mu_{\mathrm{i}}\right|}-\sum_{s=1}^{N} \mathrm{~T}_{\mathrm{i}}\left(\mathrm{x}_{\mathrm{s}}, \theta^{\mathrm{l}}\right) \frac{\left[2\left(\mathrm{x}_{\mathrm{s}}-\mu_{\mathrm{i}}\right)\right]}{\mathrm{r}^{2} \sigma_{i}{ }^{2}+\left(\mathrm{x}_{\mathrm{s}}-\mu_{\mathrm{i}}\right)^{2}}=0
\end{gathered}
$$

where

$$
\mathrm{T}_{\mathrm{i}}\left(\mathrm{x}_{\mathrm{s}}, \theta^{\mathrm{l}}\right)=\frac{\alpha_{\mathrm{i}}^{1+1} \mathrm{f}_{\mathrm{i}}\left(\mathrm{x}_{\mathrm{s}}, \theta^{1}\right)}{\sum_{\mathrm{i}=1}^{\mathrm{k}} \alpha_{\mathrm{i}}^{1+1} \mathrm{f}_{\mathrm{i}}\left(\mathrm{x}_{\mathrm{s}}, \theta^{\mathrm{l}}\right)}
$$

For For updating $\sigma_{\mathrm{i}}^{2}$ differentiate $\mathrm{Q}\left(\theta ; \theta^{\mathrm{l}}\right)$ w.r.t $\sigma_{\mathrm{i}}{ }^{2}$ and equate to 0 


$$
\begin{aligned}
& \frac{\partial}{\partial \sigma_{\mathrm{i}}{ }^{2}} \mathrm{Q}\left(\theta ; \theta^{\mathrm{l}}\right)=0 \\
& \frac{\partial}{\partial \sigma_{i}^{2}}\left[\sum_{\mathrm{i}=1}^{\mathrm{k}} \sum_{\mathrm{s}=1}^{\mathrm{N}} \mathrm{T}_{\mathrm{i}}\left(\mathrm{x}_{\mathrm{S}}, \theta^{l}\right)\left[\log \left[\left(\frac{\left(r^{2}+\frac{(x-\mu)^{2}}{\sigma^{2}}\right)^{r} e^{-\left|\frac{x-\mu}{\sigma}\right|}}{2 \sigma \sum_{k=0}^{r}\left(\begin{array}{l}
r \\
k
\end{array}\right) r^{2(r-k)}\left(\int_{\frac{a-\mu}{\sigma}}^{\frac{b-\mu}{\sigma}} x^{2 k} e^{-|x|} d x\right)}\right)\right]\right]+\log \left(\alpha_{\mathrm{i}}{ }^{1}\right)\right]=0
\end{aligned}
$$

This implies

$$
\begin{array}{r}
\frac{\partial}{\partial \sigma_{i}^{2}}\left[\sum_{\mathrm{i}=1}^{\mathrm{k}} \sum_{s=1}^{\mathrm{N}} \mathrm{T}_{\mathrm{i}}\left(\mathrm{x}_{\mathrm{s}}, \theta^{l}\right)\left[\log \left[\left(\left(r^{2}+\frac{\left(\mathrm{x}_{\mathrm{s}}-\mu_{\mathrm{i}}\right)^{2}}{\sigma_{i}^{2}}\right)^{r}\right)\right]-\left|\frac{x_{s}-\mu_{\mathrm{i}}}{\sigma_{i}}\right|-\log \left(\sigma_{i}\right)-\log \left(\sum_{k=0}^{r}\left(\begin{array}{l}
r \\
k
\end{array}\right) r^{2(r-k)}\left(\int_{\frac{a-\mu}{\sigma}}^{\frac{b-\mu}{\sigma}} x^{2 k} e^{-|x|} d x\right)\right]+\log \left(\alpha_{\mathrm{i}}^{\mathrm{l}}\right)\right]\right. \\
=0
\end{array}
$$

This implies

$$
\sum_{s=1}^{\mathrm{N}}\left[\frac{\left(\mathrm{x}_{\mathrm{s}}-\mu_{\mathrm{i}}\right)^{2}}{\left(r^{2} \sigma_{i}^{2}+\left(\mathrm{x}_{\mathrm{s}}-\mu_{\mathrm{i}}\right)^{2}\right) \sigma_{i}^{2}}-\left|\frac{x_{s}-\mu_{\mathrm{i}}}{2 \sigma_{i}{ }^{3}}\right|-\frac{1}{2 \sigma_{i}^{2}}-\frac{\sum_{k=0}^{r}\left(\begin{array}{l}
r \\
k
\end{array}\right) r^{2(r-k)}\left[\left(\frac{b-\mu_{\mathrm{i}}}{\sigma_{i}}\right)^{2 k} \cdot \frac{b-\mu_{\mathrm{i}}}{\sigma_{i}^{3}} \cdot e^{-\left|\frac{b-\mu}{\sigma}\right|}-\left(\frac{a-\mu_{\mathrm{i}}}{\sigma_{i}}\right)^{2 k} \cdot \frac{a-\mu_{\mathrm{i}}}{\sigma_{i}{ }^{3}} \cdot e^{-\left|\frac{a-\mu}{\sigma}\right|}\right]}{\sum_{k=0}^{r}\left(\begin{array}{l}
r \\
k
\end{array}\right) r^{2(r-k)}\left(\int_{\frac{a-\mu}{\sigma}}^{\frac{b-\mu}{2 k}} e^{2 k} e^{-|x|} d x\right.}\right] \mathrm{T}_{\mathrm{i}}\left(\mathrm{x}_{s}, \theta^{1}\right)=0
$$

where

$$
\mathrm{T}_{\mathrm{i}}\left(\mathrm{x}_{\mathrm{s}}, \theta^{\mathrm{l}}\right)=\frac{\alpha_{\mathrm{i}}^{1+1} \mathrm{f}_{\mathrm{i}}\left(\mathrm{x}_{\mathrm{s}}, \theta^{\mathrm{l}}\right)}{\sum_{\mathrm{i}=1}^{\mathrm{k}} \alpha_{\mathrm{i}}^{1+1} \mathrm{f}_{\mathrm{i}}\left(\mathrm{x}_{\mathrm{s}}, \theta^{\mathrm{l}}\right)}
$$

The parameter $\alpha_{\mathrm{i}}$ and the model parameters $\mu_{\mathrm{i}}$ and $\sigma_{\mathrm{i}}{ }^{2}$ have to be initialized for EM algorithm. The value for $\alpha_{\mathrm{i}}=$ $1 / \mathrm{k}$ where $\mathrm{k}$ is the number of image regions. The model parameters are obtained from hierarchical clustering (S.C. Johnson (1967)) and moment method of estimation. The truncation points $\mathrm{a}$ and $\mathrm{b}$ are estimated with minimum and maximum values of pixel intensities of the image.

solving the equations 13 and 15 we can get the final estimates of the parameters $\mu_{\mathrm{i}}$ and $\sigma_{\mathrm{i}}^{2}$.

C. Initialization of the Parameters By Hierarchical The shape parameter $\mathrm{r}$ can be estimated by sample kurtosis using the following equation clustering algorithm

$$
\begin{aligned}
& \beta_{2}=\frac{\sum_{\mathrm{k}=0}^{\mathrm{r}}\left(\begin{array}{l}
\mathrm{r} \\
\mathrm{k}
\end{array}\right) \mathrm{r}^{2(\mathrm{r}-\mathrm{k})} \sum_{\mathrm{q}=0}^{4}\left(\begin{array}{l}
4 \\
\mathrm{q}
\end{array}\right)\left(\mu-\mu_{1}^{1}\right)^{4-\mathrm{q}} \sigma^{\mathrm{q}}\left[\begin{array}{c}
(-1)^{2 \mathrm{k}+\mathrm{q}} \gamma\left[(2 \mathrm{k}+\mathrm{q}+1),-\left(\frac{\mathrm{a}-\mu}{\sigma}\right)\right]+ \\
\gamma\left[(2 \mathrm{k}+\mathrm{q}+1),\left(\frac{\mathrm{b}-\mu}{\sigma}\right)\right]
\end{array}\right]}{\left[\sum_{\mathrm{k}=0}^{\mathrm{r}}\left(\begin{array}{l}
\mathrm{r} \\
\mathrm{k}
\end{array}\right) \mathrm{r}^{2(\mathrm{r}-\mathrm{k})} \sum_{\mathrm{q}=0}^{4}\left(\begin{array}{l}
2 \\
\mathrm{q}
\end{array}\right)\left(\mu-\mu_{1}^{1}\right)^{2-\mathrm{q}} \sigma^{\mathrm{q}}\left[\begin{array}{c}
(-1)^{2 \mathrm{k}+q} \gamma\left[(2 \mathrm{k}+\mathrm{q}+1),-\left(\frac{\mathrm{a}-\mu}{\sigma}\right)\right]+ \\
\gamma\left[(2 \mathrm{k}+\mathrm{q}+1),\left(\frac{\mathrm{b}-\mu}{\sigma}\right)\right]
\end{array}\right]\right]^{2}} \\
& \sum_{\mathrm{k}=0}^{\mathrm{r}}\left(\begin{array}{l}
\mathrm{r} \\
\mathrm{k}
\end{array}\right) \mathrm{r}^{2(\mathrm{r}-\mathrm{k})} \gamma\left[(2 \mathrm{k}+1),-\left(\frac{\mathrm{a}-\mu}{\sigma}\right)\right]+\gamma\left[(2 \mathrm{k}+1),\left(\frac{\mathrm{b}-\mu}{\sigma}\right)\right] \\
& \overline{\mathrm{x}}=\mu+\frac{\sum_{\mathrm{k}=0}^{\mathrm{r}}\left(\begin{array}{l}
\mathrm{r} \\
\mathrm{k}
\end{array}\right) \mathrm{r}^{2(\mathrm{r}-\mathrm{k})}\left[\gamma\left[(2 \mathrm{k}+2),\left(\frac{\mathrm{b}-\mu}{\sigma}\right)\right]-\gamma\left[(2 \mathrm{k}+2),-\left(\frac{\mathrm{a}-\mu}{\sigma}\right)\right]\right]}{\sum_{\mathrm{k}=0}^{\mathrm{r}}\left(\begin{array}{l}
\mathrm{r} \\
\mathrm{k}
\end{array}\right) \mathrm{r}^{2(\mathrm{r}-\mathrm{k})}\left[\gamma\left[(2 \mathrm{k}+1),\left(\frac{\mathrm{b}-\mu}{\sigma}\right)\right]-\gamma\left[(2 \mathrm{k}+1),-\left(\frac{\mathrm{a}-\mu}{\sigma}\right)\right]\right]}
\end{aligned}
$$

and $\sigma^{2}=$

$$
\frac{\frac{1}{\mathrm{n}} \sum_{\mathrm{q}=0}^{2}\left(\begin{array}{l}
2 \\
\mathrm{q}
\end{array}\right)\left(\mu-\mu_{1}^{1}\right)^{2-\mathrm{q}} \sigma^{\mathrm{q}}\left[\begin{array}{c}
(-1)^{\mathrm{q}} \gamma\left[(\mathrm{q}+1),-\left(\frac{\mathrm{a}-\mu}{\sigma}\right)\right]+(-1)^{\mathrm{q}} \gamma\left[(\mathrm{q}+3),-\left(\frac{\mathrm{a}-\mu}{\sigma}\right)\right] \\
+\gamma\left[(\mathrm{q}+1),-\left(\frac{\mathrm{b}-\mu}{\sigma}\right)\right]+\gamma\left[(\mathrm{q}+3),-\left(\frac{\mathrm{b}-\mu}{\sigma}\right)\right]
\end{array}\right]}{\sum_{\mathrm{k}=0}^{\mathrm{r}}\left(\begin{array}{l}
\mathrm{r} \\
\mathrm{k}
\end{array}\right) \mathrm{r}^{2}(\mathrm{r}-\mathrm{k})\left[\gamma\left[(2 \mathrm{k}+1),-\left(\frac{\mathrm{a}-\mu}{\sigma}\right)\right]-\gamma\left[(2 \mathrm{k}+1),\left(\frac{\mathrm{b}-\mu}{\sigma}\right)\right]\right]}
$$

Solving equations 16,17 and 18 simultaneously by Newton Raphson Method the parameters $\mu_{\mathrm{i}}$ and $\sigma_{\mathrm{i}}^{2}$ are obtained. With these initial estimates final estimates are obtained through EM algorithm as illustrated in section B.

\section{Segmentation Algorithm}

I.J. Image, Graphics and Signal Processing, 2017, 1, 41-49 
Once the final estimates of parameters are obtained, next step is to segment the image by assigning the pixels to segments. This is achieved through the following segmentation algorithm.

Step 1: Attain the number of image segments using hierarchical clustering algorithm.

Step2: Obtain the initial estimates of model parameters using hierarchical clustering.

Step 3: Using the EM algorithm obtain the refined estimates of the model parameters $\alpha_{i}, \mu_{i}, \sigma_{i}^{2}$ for $i=1,2 \ldots . k$.

Step 4: Assign each pixel to corresponding $\mathrm{j}^{\text {th }}$ region according to maximum likelihood of the segment $\mathrm{L}_{\mathrm{j}}$. The pixel $\mathrm{z}_{\mathrm{s}}$ is assigned to the $\mathrm{j}^{\text {th }}$ segment for which $\mathrm{L}$ is maximum.

$$
\mathrm{L}=\max \left(\frac{\left(\mathrm{r}^{2}+\frac{(\mathrm{x}-\mu)^{2}}{\sigma^{2}}\right)^{\mathrm{r}} \mathrm{e}^{-\left|\frac{\mathrm{x}-\mu}{\sigma}\right|}}{2 \sigma \sum_{\mathrm{k}=0}^{\mathrm{r}}\left(\begin{array}{l}
\mathrm{r} \\
\mathrm{k}
\end{array}\right) \mathrm{r}^{2(\mathrm{r}-\mathrm{k})}\left(\int_{\frac{\mathrm{a}-\mu}{\sigma}}^{\frac{\mathrm{b}-\mu}{\sigma}} \mathrm{x}^{2 \mathrm{k}} \mathrm{e}^{-|\mathrm{x}|} \mathrm{dx}\right)}\right)
$$

\section{EXPERIMENTATION AND RESULTS}

For experimentation five images randomly were taken from Berkeley image dataset (www.eecs.berkeley.edu/Research/Projects/CS/vision/bs ds/BSDS300/html/dataset/images.html). The pixel intensity values in image are chosen as feature of the image assuming that they follow generalized Laplace distribution. Image consists of $\mathrm{K}$ image regions and initial value of $\mathrm{K}$ is obtained by histogram of pixel intensities. The five images and their respective histograms are shown in Figure1.

Image
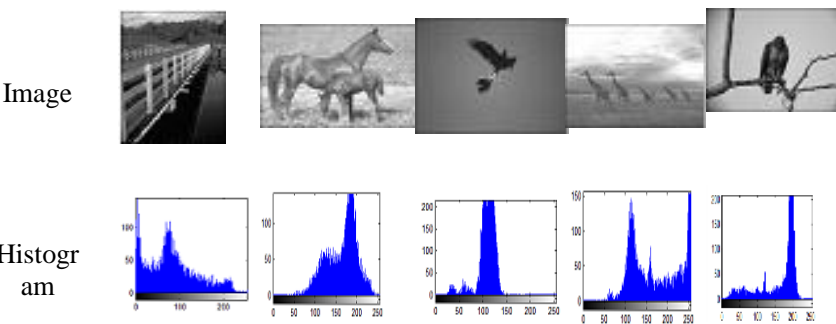

Fig.1. Images and their Histograms.

The model parameters considered are $\alpha_{\mathrm{i}}, \mu_{\mathrm{i}}$ and $\sigma_{\mathrm{i}}$ for $\mathrm{i}=1,2 \ldots \mathrm{K}$ and are obtained by the method given in section 4. Final parameters for the five images have been derived using these initial parameters and updated equations in section 3 the final parameters and presented in Tables 1,2,3,4 and 5.
Table 1. Estimation of parameters for Image1

\begin{tabular}{|c|c|c|c|c|c|c|}
\hline \multicolumn{7}{|c|}{$\begin{array}{l}\text { Estimated Values of the Parameters for Image1 } \\
\qquad \text { Number of regions }(\mathrm{K}=3)\end{array}$} \\
\hline \multirow[t]{2}{*}{ Parameters } & \multicolumn{3}{|c|}{$\begin{array}{c}\text { Estimation of Initial } \\
\text { Parameters } \\
\text { By Hierarchical } \\
\text { Clustering }\end{array}$} & \multicolumn{3}{|c|}{$\begin{array}{l}\text { Estimation of Final } \\
\text { Parameters by EM } \\
\text { Algorithm }\end{array}$} \\
\hline & $\mathrm{C} 1$ & $\mathrm{C} 2$ & C3 & $\mathrm{C} 1$ & $\mathrm{C} 2$ & C3 \\
\hline$\alpha_{\mathrm{i}}$ & 0.33 & 0.33 & 0.33 & $\begin{array}{c}- \\
0.136 \\
\end{array}$ & 0.985 & 0.150 \\
\hline$\mu_{\mathrm{i}}$ & 102.72 & 244.9 & 148.3 & 251.9 & 198.3 & 261.1 \\
\hline$\sigma_{\mathrm{i}}$ & 10.79 & 6.90 & 15.29 & 13.37 & 51.31 & 40.74 \\
\hline \multicolumn{4}{|c|}{$a=0$} & \multicolumn{3}{|c|}{$b=255$} \\
\hline
\end{tabular}

Table 2. Estimation of parameters for Image2

\begin{tabular}{|c|c|c|c|c|c|c|}
\hline \multicolumn{7}{|c|}{$\begin{array}{c}\text { Estimated Values of the Parameters for Image2 } \\
\text { Number of regions }(\mathrm{K}=3)\end{array}$} \\
\hline \multirow{3}{*}{ Parameters } & \multicolumn{3}{|c|}{$\begin{array}{c}\text { Estimation of Initial } \\
\text { Parameters } \\
\text { By Hierarchical } \\
\text { Clustering }\end{array}$} & \multicolumn{3}{c|}{$\begin{array}{c}\text { Estimation of Final } \\
\text { Parameters by EM } \\
\text { Algorithm }\end{array}$} \\
\cline { 2 - 7 } & $\mathrm{C} 1$ & $\mathrm{C} 2$ & $\mathrm{C} 3$ & $\mathrm{C} 1$ & $\mathrm{C} 2$ & $\mathrm{C} 3$ \\
\hline$\alpha_{\mathrm{i}}$ & 0.33 & 0.33 & 0.33 & 0.43 & 0.43 & 0.13 \\
\hline$\mu_{\mathrm{i}}$ & 185.37 & 151.79 & 69.28 & 190.55 & 48.16 & 111.8 \\
\hline$\sigma_{\mathrm{i}}$ & 9.42 & 8.95 & 11.13 & 15.53 & 11.30 & 18.2 \\
\hline \multicolumn{3}{|c|}{$\mathrm{a}=34$} & & & $\mathrm{~b}=254$ & \\
\hline
\end{tabular}

Table 3. Estimation of parameters for Image 3

\begin{tabular}{|c|c|c|c|c|c|c|}
\hline \multicolumn{7}{|c|}{$\begin{array}{c}\text { Estimated Values of the Parameters for Image3 } \\
\text { Number of regions }(\mathrm{K}=3)\end{array}$} \\
\hline $\begin{array}{c}\text { Paramete } \\
\text { rs }\end{array}$ & \multicolumn{3}{|c|}{$\begin{array}{c}\text { Estimation of Initial } \\
\text { Parameters } \\
\text { By Hierarchical Clustering }\end{array}$} & \multicolumn{3}{|c|}{$\begin{array}{c}\text { Estimation of Final } \\
\text { Parameters by EM } \\
\text { Algorithm }\end{array}$} \\
\cline { 2 - 7 } & $\mathrm{C} 1$ & $\mathrm{C} 2$ & $\mathrm{C} 3$ & $\mathrm{C} 1$ & $\mathrm{C} 2$ & $\mathrm{C} 3$ \\
\hline$\alpha_{\mathrm{i}}$ & 0.33 & 0.33 & 0.33 & 0.352 & -0.021 & 0.668 \\
\hline$\mu_{\mathrm{i}}$ & 144.5 & 53.46 & 180.8 & 170.5 & 49.37 & 194.8 \\
\hline$\sigma_{\mathrm{i}}$ & 7.21 & 16.03 & 4.94 & 18.53 & 18.30 & 15.24 \\
\hline \multicolumn{3}{|c|}{$\mathrm{a}=7$} & \multicolumn{3}{|c|}{$\mathrm{b}=187$} \\
\hline
\end{tabular}

Table 4. Estimation of parameters for Image4

\begin{tabular}{|c|c|c|c|c|c|c|}
\hline \multicolumn{7}{|c|}{$\begin{array}{l}\text { Estimated Values of the Parameters for Image4 } \\
\text { Number of regions }(K=3)\end{array}$} \\
\hline \multirow[t]{2}{*}{ Parameters } & \multicolumn{3}{|c|}{$\begin{array}{c}\text { Estimation of Initial } \\
\text { Parameters } \\
\text { By Hierarchical } \\
\text { Clustering }\end{array}$} & \multicolumn{3}{|c|}{$\begin{array}{c}\text { Estimation of Final } \\
\text { Parameters by EM } \\
\text { Algorithm }\end{array}$} \\
\hline & $\mathrm{C} 1$ & $\mathrm{C} 2$ & C3 & $\mathrm{C} 1$ & $\mathrm{C} 2$ & C3 \\
\hline$\alpha_{i}$ & 0.33 & 0.33 & 0.33 & 0.7666 & $\begin{array}{c}- \\
0.0018\end{array}$ & 0.2343 \\
\hline$\mu_{\mathrm{i}}$ & 242 & 34.33 & 179.79 & 193.86 & 58.16 & 159.80 \\
\hline$\sigma_{\mathrm{i}}$ & 12.63 & 5.85 & 18.36 & 10.09 & 13.70 & 20.27 \\
\hline \multicolumn{4}{|c|}{$a=30$} & \multicolumn{3}{|c|}{$\mathrm{b}=255$} \\
\hline
\end{tabular}


Table 5. Estimation of parameters for Image5

\begin{tabular}{|c|c|c|c|c|c|c|}
\hline \multicolumn{7}{|c|}{$\begin{array}{c}\text { Estimated Values of the Parameters for Image5 } \\
\text { Number of regions(K=3) }\end{array}$} \\
\hline Parameters & \multicolumn{3}{|c|}{$\begin{array}{c}\text { Estimation of Initial } \\
\text { Parameters } \\
\text { By Hierarchical Clustering }\end{array}$} & $\begin{array}{c}\text { Estimation of Final } \\
\text { Parameters by EM } \\
\text { Algorithm }\end{array}$ \\
\hline & $\mathrm{C} 1$ & $\mathrm{C} 2$ & $\mathrm{C} 3$ & $\mathrm{C} 1$ & $\mathrm{C} 2$ & $\mathrm{C} 3$ \\
\hline$\alpha_{\mathrm{i}}$ & 0.33 & 0.33 & 0.33 & -0.18 & 1.23 & -0.04 \\
\hline$\mu_{\mathrm{i}}$ & 110.91 & 190.53 & 51.08 & 24.05 & 116.42 & 72.04 \\
\hline$\sigma_{\mathrm{i}}$ & 14.39 & 14.82 & 18.62 & 10.53 & 14.30 & 13.26 \\
\hline \multicolumn{3}{|c|}{$\mathrm{a}=5$} & & \multicolumn{4}{c|}{$\mathrm{b}=231$} \\
\hline
\end{tabular}

Substituting the final estimates of the model parameters the probability density function of the pixel intensities in each image are estimated.

The estimated probability density function of the pixel intensities of the image 1 is

$$
\begin{aligned}
& \frac{1}{\mathrm{f}\left(\mathrm{x}, \theta^{1}\right)=}\left(1+\frac{(x-251.93)^{2}}{13.37^{2}}\right)^{r} e^{-\left|\frac{x-251.93}{13.37}\right|}+ \\
& \frac{1}{102.62}\left(1+\frac{(x-198.30)^{2}}{51.31^{2}}\right)^{r} e^{-\left|\frac{x-198.30}{51.31}\right|}+ \\
& \frac{1}{81.48}\left(1+\frac{(x-261.12)^{2}}{40.74^{2}}\right) e^{-\left|\frac{x-261.12}{40.74}\right|}
\end{aligned}
$$

The estimated probability density function of the pixel intensities of the image 2 is

$$
\begin{gathered}
\mathrm{f}\left(\mathrm{x}, \theta^{1}\right)= \\
\frac{1}{31.06}\left(1+\frac{(x-190.55)^{2}}{15.53^{2}}\right)^{r} e^{-\left|\frac{x-190.55}{15.53}\right|}+ \\
\frac{1}{22.6}\left(1+\frac{(x-48.16)^{2}}{11.30^{2}}\right)^{r} e^{-\left|\frac{x-48.16}{11.30}\right|}+ \\
\frac{1}{36.4}\left(1+\frac{(x-111.8)^{2}}{18.2^{2}}\right)^{r} e^{-\left|\frac{x-111.8}{18.2}\right|}
\end{gathered}
$$

The estimated probability density function of the pixel intensities of the image 3 is

$$
\begin{gathered}
\mathrm{f}\left(\mathrm{x}, \theta^{1}\right)= \\
\frac{1}{37.06}\left(1+\frac{(x-170.55)^{2}}{18.53^{2}}\right)^{r} e^{-\left|\frac{x-170.55}{18.53}\right|}+ \\
\frac{1}{36.6}\left(1+\frac{(x-49.37)^{2}}{18.3^{2}}\right)^{r} e^{-\left|\frac{x-49.37}{18.30}\right|}+ \\
\frac{1}{30.48}\left(1+\frac{(x-194.80)^{2}}{15.24^{2}}\right)^{r} e^{-\left|\frac{x-194.8}{15.24}\right|}
\end{gathered}
$$

The estimated probability density function of the pixel intensities of the image 4 is

$$
\begin{aligned}
& \mathrm{f}\left(\mathrm{x}, \theta^{1}\right)= \\
& \frac{1}{20.18}\left(1+\frac{(x-193.8)^{2}}{10.09^{2}}\right)^{r} e^{-\left|\frac{x-193.8}{10.09}\right|}+ \\
& \frac{1}{27.4}\left(1+\frac{(x-58.16)^{2}}{13.7}\right)^{r} e^{-\left|\frac{x-58.16}{13.7}\right|}+ \\
& \frac{1}{40.54}\left(1+\frac{(x-159.80)^{2}}{20.27}\right)^{r} e^{-\left|\frac{x-159.80}{20.27}\right|}
\end{aligned}
$$

The estimated probability density function of the pixel intensities of the image 5 is

$$
\begin{gathered}
\mathrm{f}\left(\mathrm{x}, \theta^{1}\right)= \\
\frac{1}{21.06}\left(1+\frac{(x-24.05)^{2}}{10.53^{2}}\right)^{r} e^{-\left|\frac{x-24.05}{10.53}\right|}+ \\
\frac{1}{28.60}\left(1+\frac{(x-116.42)^{2}}{14.30^{2}}\right)^{r} e^{-\left|\frac{x-116.42}{18.30}\right|}+ \\
\frac{1}{26.52}\left(1+\frac{(x-72.04)^{2}}{13.26^{2}}\right)^{r}
\end{gathered}
$$

Using the probability density function and segmentation algorithm, image segmentation is performed for following images. The original images and segmented images are shown in Figure 2.

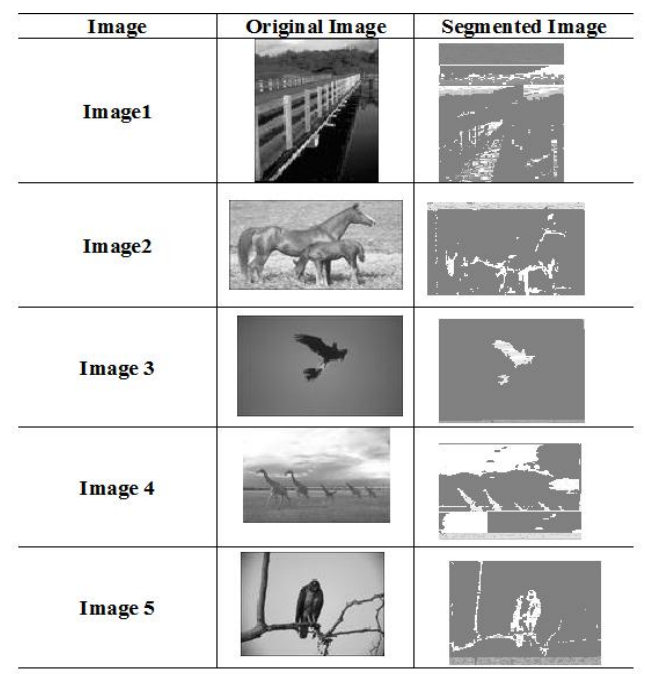

Fig.2. Original Image and Segmented Image

\section{Performance Measures}

Once the image segmentation has been performed, its performance has been measured by calculating the performance metrics like probabilistic rand index(PRI) given by Unnikrishnan R et al(2007), global consistency error(GCE) given BY Martin D. and et al and variation of information (VOI) given by Meila M(2005). The standard criteria for metrics is that PRI and GCE values must lie in range 0 to 1 while VOI can take value as big as possible. The performance metrics for image segmentation method based on doubly truncated generalized mixture model using hierarchical clustering 
(DTGLMM-H) is shown in table 6 and compared with segmentation method based on GMM, GLMM using Kmeans (GLMM-K), GLMM using Hierarchical clustering (GLMM-H) and doubly truncated generalized mixture model using K-means algorithm (DTGLMM-K).

From table 6 it is observed that the proposed method satisfies the standard criteria for the performance measures PRI, GCE and VOI. The performance metrics are compared with GMM, GLMM-K, GLMM-H and DTGLMM-K and presented in Figure 3.

The image can also be reconstructed using the developed algorithm. Different quality metrics like image fidelity mean square error and image quality can be computed to study the performance of image quality. The metrics have been calculated and shown in Table7.

From Table 7 it can be seen that the proposed method DTGLMM-H has performed well when compared with other methods.

The original images and reconstructed images using developed segmentation algorithm are presented in figure 4.

Table 6. Performance Measures

\begin{tabular}{|c|c|c|c|c|}
\hline \multirow{2}{*}{ Image } & \multirow{2}{*}{ Method } & \multicolumn{3}{|c|}{ Performance Measures } \\
\hline & & PRI & GCE & VOI \\
\hline \multirow{5}{*}{ Image 1 } & GMM & 0.94 & 0.72 & 2.84 \\
\hline & GLMM-K & 0.98 & 0.73 & 5.31 \\
\hline & GLMM-H & 0.99 & 0.68 & 2.25 \\
\hline & DTGLMM-K & 0.99 & 0.11 & 0.83 \\
\hline & DTGLMM-H & 0.9988 & 0.1150 & 0.8164 \\
\hline \multirow{5}{*}{ Image 2} & GMM & 0.96 & 0.78 & 2.55 \\
\hline & GLMM-K & 0.98 & 0.75 & 5.22 \\
\hline & GLMM-H & 0.98 & 0.75 & 2.23 \\
\hline & DTGLMM-K & 0.99 & 0.10 & 0.87 \\
\hline & DTGLMM-H & 0.9975 & 0.1065 & 0.8749 \\
\hline \multirow{5}{*}{ Image 3} & GMM & 0.97 & 0.77 & 2.32 \\
\hline & GLMM-K & 0.97 & 0.71 & 5.20 \\
\hline & GLMM-H & 0.98 & 0.75 & 1.48 \\
\hline & DTGLMM-K & 0.99 & 0.10 & 0.85 \\
\hline & DTGLMM-H & 0.9964 & 0.1029 & 0.8740 \\
\hline \multirow{5}{*}{ Image4 } & GMM & 0.97 & 0.69 & 2.23 \\
\hline & GLMM-K & 0.97 & 0.72 & 2.88 \\
\hline & GLMM-H & 0.98 & 0.66 & 1.45 \\
\hline & DTGLMM-K & 0.99 & 0.035 & 0.25 \\
\hline & DTGLMM-H & 0.9980 & 0.0385 & 0.2702 \\
\hline \multirow{5}{*}{ Image5 } & GMM & 0.96 & 0.77 & 2.12 \\
\hline & GLMM-K & 0.98 & 0.75 & 2.34 \\
\hline & GLMM-H & 0.97 & 0.73 & 2.02 \\
\hline & DTGLMM-K & 0.99 & 0.10 & 0.72 \\
\hline & DTGLMM-H & 0.9986 & 0.1027 & 0.7278 \\
\hline
\end{tabular}

Table 7. Segmentation Quality Metrics

\begin{tabular}{|c|c|c|c|c|}
\hline \multirow{2}{*}{ Image } & \multirow{2}{*}{ Method } & \multicolumn{3}{|c|}{ Image Quality Metrics } \\
\hline & & $\begin{array}{c}\text { Image } \\
\text { Fidelity }\end{array}$ & $\begin{array}{l}\text { Signal to } \\
\text { Noise } \\
\text { Ratio }\end{array}$ & $\begin{array}{c}\text { Image } \\
\text { Quality } \\
\text { Index }\end{array}$ \\
\hline \multirow{5}{*}{ Image 1 } & GMM & 0.99 & 0.24 & 1.0 \\
\hline & GLMM-H & 0.99 & 0.24 & 0.988 \\
\hline & GLMM-K & 0.99 & 0.419 & 0.988 \\
\hline & DTGLMM-K & .99 & 0.34 & .99 \\
\hline & DTGLMM-H & 0.99 & 0.38 & 0.9943 \\
\hline \multirow{5}{*}{ Image 2} & GMM & 0.99 & 2.01 & 0.99 \\
\hline & GLMM-K & 0.99 & 0.41 & 0.99 \\
\hline & GLMM-H & 0.99 & 2.03 & 0.99 \\
\hline & DTGLMM-K & .99 & 3.34 & .99 \\
\hline & DTGLMM-H & 0.9985 & 4.21 & 0.9915 \\
\hline \multirow{5}{*}{ Image 3} & GMM & 0.98 & 1.45 & 0.98 \\
\hline & GLMM-K & 0.99 & 0.03 & 0.99 \\
\hline & GLMM-H & 0.99 & 1.45 & 0.99 \\
\hline & DTGLMM-K & 0.99 & 2.25 & .98 \\
\hline & DTGLMM-H & 0.9965 & 2.53 & 0.9836 \\
\hline \multirow{5}{*}{ Image4 } & GMM & .99 & 2.23 & 1.0 \\
\hline & GLMM-K & .98 & 4.4 & .998 \\
\hline & GLMM-H & .99 & 2.69 & 1.0 \\
\hline & DTGLMM-K & 1.0 & 4.5 & .99 \\
\hline & DTGLMM-H & 1.0 & 4.0 & 0.9998 \\
\hline \multirow{5}{*}{ Image 5 } & GMM & .99 & 1.09 & 0.99 \\
\hline & GLMM-K & .99 & 2.67 & .99 \\
\hline & GLMM-H & .99 & 1.13 & .997 \\
\hline & DTGLMM-K & .99 & 3.77 & .99 \\
\hline & DTGLMM-H & 0.99 & 2.83 & 0.9925 \\
\hline
\end{tabular}




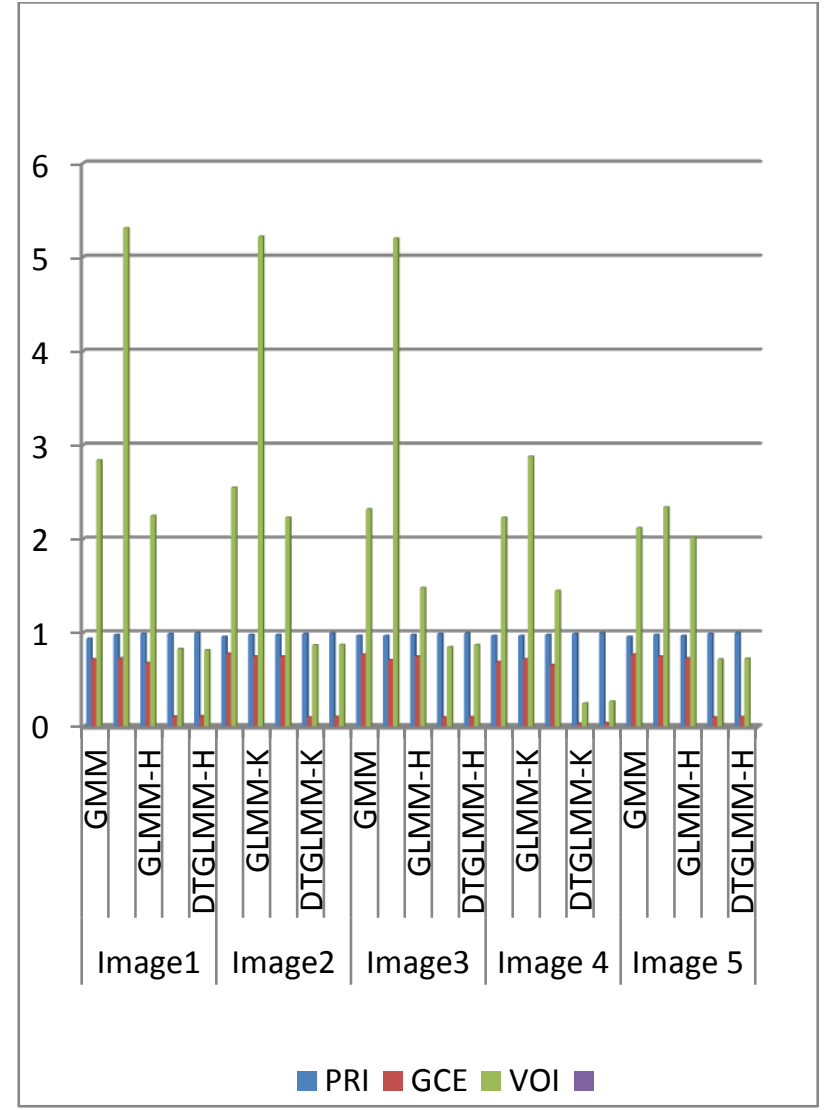

Fig.3. Comparison of Performance Metrics.

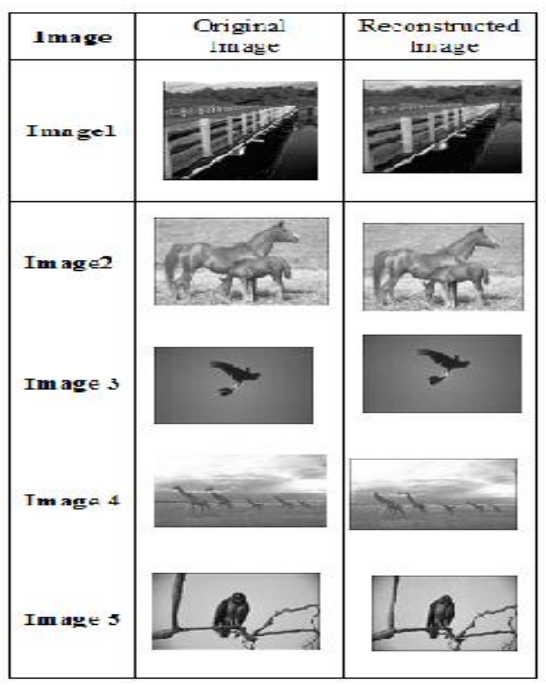

Fig.4. Original and Reconstructed Images

\section{CONCLUSION}

This paper addresses the image segmentation method based on doubly truncated generalized Laplace distribution mixture model and hierarchical clustering. The feature vector associated with the image region is characterized by truncated generalized Laplace distribution which improves Laplace and generalized laplace distributions as limiting cases. The doubly truncated generalized laplace distribution includes a spectrum of probability models which may be meso kurtic, platy kurtic, lepto kurtic, symmetric and asymmetric. The effect of truncation on probability model has a significant influence since in reality the pixel intensities are having finite range. The model parameters are estimated by deriving updated equations of the scale and location parameters. The shape parameter is estimated using sample kurtosis. The initialization of parameter is carried using hierarchical clustering for initial segmentation for whole image and moment methods of estimation. The performance of the algorithm is analyzed through experimentation on randomly chosen five images from Berkeley data set. The performance measures such as PRI, GCE and VOI revealed that this algorithm perform better than the earlier algorithms. This may be due to the effect of truncation used for modeling feature vector. The hierarchical clustering algorithm used for initialization of parameters reduces the computational complexities and convergence of the EM algorithm. The proposed algorithm is much useful for analyzing images arising at several domains of applications. It is possible to extend this image segmentation method for color images considering a 3-dimensional feature vector which will be taken up elsewhere.

\section{REFERENCES}

[1] Wang Xue-guang, et al., “An Improved Image Segmentation Algorithm Based on Two-Dimensional Otsu Method", "Information Science Letters An International Journal", No. 2, 77-83,2012.

[2] Li Zhengzhou, et al., "Gray-scale Edge Detection and Image Segmentation Algorithm Based on Mean Shift", "Telkomnika Indonesian Journal of Electrical Engineering”, Vol.11, No.3, pp. 1414 -1421, March 2013.

[3] Mahbubun Nahar, et al., "An Improved Approach for Digital Image Edge Detection", "International Journal of Recent Development in Engineering and Technology", Vol.2, Issue3, 2014

[4] Razieh Akhavan, et al., "A Novel Retinal Blood Vessel Segmentation Algorithm using Fuzzy segmentation", "International Journal of Electrical and computer Engineering (IJECE)”, vol 4 No 4, pp 561-572, 2014

[5] Suetens, et al., "Model-Based Image Segmentation: Methods and Applications", "Lecture Notes in Medical Informatics" Vol.44, pp3-24.

[6] Yunjie Chen, et al.,"An Improved Gaussian Mixture Model based on NonLocal Information for Brain MR Images Segmentation”, "International Journal of Signal Processing, Image Processing and Pattern Recognition" Vol.7, No.4, pp.187-194, 2014.

[7] Karim Kalti, et al., "Image Segmentation by Gaussian Mixture Models and Modified FCM Algorithm", "The International Arab Journal of Information Technology", Vol. 11, No. 1, January 2014.

[8] Zhaoxia $\mathrm{Fu}$, et al., "Color Image Segmnetation using Gaussian Mixture Model and EM Algorithm", "Multimedia and Signal Processing CCIS 346 Springer", pp 61-66, 2012.

[9] Srinivas Yerramalle, et al., "Unsupervised image classification using finite truncated Gaussian mixture model", "Journal of Ultra Science for Physical Sciences", Vol.19, No.1, pp 107-114.

[10] Jyothirmayi T et al." "Studies on Image Segmentation Integrating Generalized Laplace Mixture Model and 
Hierarchichal Clustering", "International Journal of Computer Applications", Vol 128, No 12 pp 7-13, 2015.

[11] M.Vamsi Krishna, et al., "Bivariate Gaussian Mixture Model Based Segmentation for Effective Identification of Sclerosis in Brain MRI Images", "International Journal of Engineering and Technical Research", Vol 3 Issue 1 pp 151-154, 2015.

[12] Hanze Zhang, et al., "Finite Mixture Models and their Applications: A Review", "Austin Biometrics and Biostatistics", vol 2 issue 1, 2015.

[13] Mclanchlan G. et al., "The EM algorithm and Extensions", John Wiley and sons, New York-1997.

[14] Johnson S.C "A Tutorial on Clustering Algorithms", http://home.dei.polimi.it/matteucc/Clustering/tutorial_htm 1/hierarchical.html.

[15] Martin D. , et al., " A database of human segmented natural images and its application to evaluating segmentation algorithms and measuring ecological statistics," in proc. 8th Int. Conf. Computer vision, vol.2, pp.416-423,2001.

[16] Norman L.Johnson, Kortz and Balakrishnan, "Continuous Univariate distributions"Volume-I, John Wiley and Sons Publications,Newyork, 1994.

[17] Nikita Sharma, et al., " Colour Image Segmentation Techniques And Issues: An Approach", "International Journal of Scientific \& Technology Research", Volume 1, Issue 4, May 2012.

[18] T.Yamazaki, "Introduction of EM algorithms into color image segmentation", in proceedings of ICIRS'98,pp368371,1998

[19] M. Seshashayee et al.,, "Studies on Image Segmentation method Based on a New Symmetric Mixture Model with K - Means", "Global journal of Computer Science and Technology", Vol.11, No.18, pp.51-58,2011.

[20] Jahne (1995), "A Practical Hand Book on Image segmentation for Scientific Applications, CRC Press.

\section{Authors' Profiles}

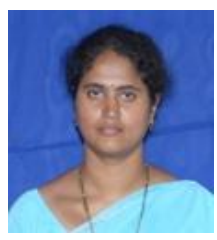

T. Jyothirmayi is presently working as Assistant Professor in the department of Computer Science and Engineering, GIT, GITAM University, Visakhapatnam. She presented research papers in national and international conferences and journals of good repute. She guided several students for Project work in department of Computer Science Engineering. Her current research interests include image processing and data mining.

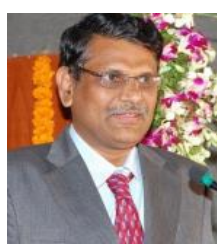

Dr. K. Srinivasa Rao is presently working as Professor, Department of Statistics, Andhra University, Visakhapatnam. He is elected chief editor of Journal of ISPS and elected Vice- President of Operation Research of India. He guided 52 students for Ph.D in Statistics, Mathematics, Computer Science Electronics and Communications Engineering, Industrial Engineering and Operations Research. He published more than 130 research papers in National and International journals with high reputation. His research interests are Image Processing, Communication Systems, Data Mining and Stochastic models.

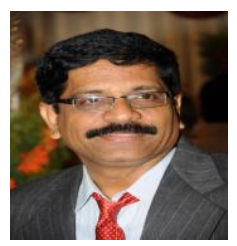

Dr. Peri. Srinivasa Rao is presently working as Professor in the Department of Computer Science and Systems Engineering, Andhra University, Visakhapatnam. He got his Ph.D degree from Indian Institute of Technology, Kharagpur in Computer Science in 1987. He published several research papers and delivered invited lectures at various conferences, seminars and workshops. He guided a number of students for their Ph.D and M.Tech degrees in Computer Science and Engineering and Information Technology. His current research interests are Image Processing, Communication networks, Data Mining and Computer Morphology.

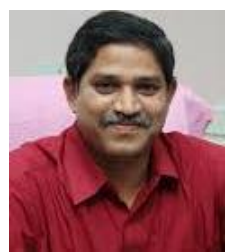

Dr. Ch. Satyanarayana is currently Professor in Computer Science and Engineering Department at Jawaharlal Nehru Technological University -Kakinada He has 17 years of experience. His area of interest is on Image processing, Database Management, Network security. He published more than 45 research papers in international journals and guided M.Tech and Ph.D students.

How to cite this paper: T.Jyothirmayi, K.Srinivasa Rao, P.Srinivasa Rao, Ch.Satyanarayana,"Performance Evaluation of Image Segmentation Method based on Doubly Truncated Generalized Laplace Mixture Model and Hierarchical Clustering", International Journal of Image, Graphics and Signal Processing(IJIGSP), Vol.9, No.1, pp.41-49, 2017.DOI: $10.5815 /$ ijigsp.2017.01.06 